\title{
Cancer gene related characterization of patterns and point of recurrence after resection of colorectal liver metastases
}

\author{
Hauke Lang $^{1 \#}$, Janine Baumgart ${ }^{1 \#}$, Wilfried Roth ${ }^{2}$, Markus Moehler ${ }^{3}$, Michael Kloth ${ }^{2}$ \\ ${ }^{1}$ Department of General, Visceral and Transplantation Surgery, Universitätsmedizin Mainz, Mainz, Germany; ${ }^{2}$ Department of Pathology, \\ Universitätsmedizin Mainz, Mainz, Germany; ${ }^{3}$ Department of Gastroenterology and Hepatology, Universitätsmedizin Mainz, Mainz, Germany \\ Contributions: (I) Conception and design: H Lang, J Baumgart; (II) Administrative support: W Roth, M Moehler; (III) Provision of study materials \\ or patients: J Baumgart, M Moehler, M Kloth; (IV) Collection and assembly of data: J Baumgart, M Kloth; (V) Data analysis and interpretation: H \\ Lang, J Baumgart, M Kloth; (VI) Manuscript writing: All authors; (VII) Final approval of manuscript: All authors. \\ "These authors contributed equally to this work. \\ Correspondence to: Prof. Dr. Hauke Lang, MA, FACS. Department of General, Visceral and Transplantation Surgery, Universitätsmedizin Mainz, \\ Langenbeckstraße 1, 55131 Mainz, Germany. Email: hauke.lang@unimedizin-mainz.de.
}

Background: Outcome after resection of CRLM is hampered by a high rate of recurrence. There are little data about the role of cancer related genes and their mutations in this scenario. The aim of our analysis was to assess the predictive power of cancer-related genes and their mutations on risk for and distribution of recurrence and the time of occurrence after resection of colorectal liver metastases (CRLM).

Methods: We included 130 patients with 167 liver resections. The work-up consisted of the analysis of a total of 720 cancer-related genes by next-generation sequencing (NGS). Results were correlated with the patterns and time of recurrence and survival.

Results: At the time of analysis, 89/130 patients (68\%) had developed recurrence. This included liver only recurrence in $52 \%$, lung only recurrence in $11 \%$ and disseminated disease in $37 \%$ of cases. In univariate analysis, alterations in the RAS/RAF pathway and in the SMAD family had significant predictive power for the time of recurrence $(\mathrm{P}<0.0001)$ whereas single mutations did not reach statistical significance in multivariate analysis. Mutations of PIK3CA were associated with a better prognosis and a later occurrence of relapse. A recurrence risk score ( $r-R S)$ based on mutations in these cancer related genes is predictive of the time of recurrence.

Conclusions: In conclusion, mutations in the RAS/RAF pathway and the SMAD family are risk factors for early recurrence. Mutations of PIK3CA are associated with a lower risk for recurrence after resection of CRLM. Cancer related genes and their mutations do not correlate with patterns of recurrence but are predictive for the timely onset of recurrence.

Keywords: Colorectal liver metastases (CRLM); liver resection; next generation sequencing (NGS); recurrence after liver resection

Submitted Jan 19, 2021. Accepted for publication May 21, 2021.

doi: 10.21037/atm-21-292

View this article at: https://dx.doi.org/10.21037/atm-21-292

\section{Introduction}

The mainstay of treatment of colorectal liver metastases (CRLM) is a synergy of hepatic resection and systemic chemotherapy, often in combination with targeted therapy (1-4). Nevertheless, more than $50 \%$ of patients develop disease recurrence after potentially curative liver resection $(5,6)$.
However, the impressive progress in surgery and in medical therapy has improved outcome of recurrent CRLM as well $(3,7)$. Nowadays, treatment options for this scenario range from systemic over potentially curative approaches by repeated resections to even liver transplantation, depending on the extent and distribution of tumor relapse (7-11). Therefore, 
a better understanding of the patterns of recurrent disease is essential for patient selection and stratification of therapy.

In recent years, analyses of cancer related genes and their mutations, mainly referring to the RAS/RAF pathway, the SMAD family as well as TP53 and PIK3CA, have broadened the spectrum of markers predictive for outcome (12-15). The influence of cancer related genes and their mutations on tumor relapse after resection of CRLM is not yet well investigated. The aim of our analysis was to assess the predictive power of cancer related genes and their mutations on rates, time of occurrence as well as on distribution of recurrence after liver resection for CRLM.

We present the following article in accordance with the STROBE reporting checklist (available at https://dx.doi. org/10.21037/atm-21-292).

\section{Methods}

\section{Study design}

We included 223 resections in 158 patients in the current analysis. The full work up contained clinical and perioperative data from our prospective institutional liver database and a complete analysis of 720 cancer related genes and their copy number changes by next-generation sequencing (NGS). Postoperative follow-up to detect recurrent disease included a computed tomography (CT) scan of the chest and a CTscan or magnetic resonance imaging (MRI) of the abdomen every 3 months within the first year. Thereafter, the time interval was extended to 6 months.

Results of molecular profiling were correlated with survival data as well as with the patterns of recurrence, including rates, time and distribution of recurrence.

The study was approved by the local ethic committee [Ethikkomitee der Universitätsmedizin Mainz; 837.141.13(8826-F)]. The study conforms to the provisions of the Declaration of Helsinki (as revised in 2013). All patients signed an informed consent before participating the study.

\section{Genomic analysis}

Sequencing, mutation calls and copy number analyses were performed as described previously (16).

\section{Statistics}

Patients with extrahepatic disease at the time of liver surgery and with R2-resection margins were excluded from the analysis.

Recurrence was divided in 3 clinical groups: liver only, lung only and disseminated tumor spread. Recurrencefree survival (RFS) was calculated from the date of liver resection until any occurrence of tumor disease.

Statistical analyses were performed in the $\mathrm{R}$ statistical computing environment. Parameters that were significant on univariate analysis were entered in a multivariate Cox analysis. $\mathrm{P}$ values $<0.05$ were considered significant.

\section{Results}

\section{Study population}

Out of the 158 patients we excluded 28 patients, 26 due to extrahepatic disease at the time of liver surgery and 2 patients with $\mathrm{R} 2$-resections. In another 28 patients having a two-stage procedure (TSH \pm PVE/PVL, ALPPS) we looked at step 1 and 2 operations as one procedure. Summarized, data of 130 patients and 167 liver resections were eligible for the overall analysis. Patients' characteristics and operative data are listed in Table 1.

\section{Survival and recurrence}

The median follow-up after liver surgery was 39.4 months and the median overall survival (OS) of the entire cohort was 41.2 months. There was one in-hospital death after 34 days due to septicaemia after endoscopic intervention at the bile duct.

Tumor recurrence was observed in 89 patients (68\%). The patterns of recurrence were as following: liver only in 46/89 (52\%), lung only in 10/89 (11\%) and disseminated tumor spread in 33/89 cases (37\%) (Figure 1). Disseminated disease included 4 patients with simultaneous hepatic and pulmonary metastases, hepatic metastases and the involvement of at least one more organ system in another 13 patients and extrahepatic recurrence in 16 patients. The median RFS of the entire cohort was 16.8 months.

A subgroup of 6 patients was prominent by having at least 2 and up to 4 repeated liver only recurrences being eligible for liver surgery each time (3 patients with 3 liver resections, 1 patient with 4 liver resections and 2 patients with 5 liver resections, each).

\section{Extended molecular profiling}

We analysed recurrent somatic mutations and copy number 
Table 1 Clinical characteristics and operative data

\begin{tabular}{|c|c|}
\hline Characteristics & Number \\
\hline Patients & 130 \\
\hline Sex, male/female & $80 / 50$ \\
\hline Age (years), median (range) & $60.5[36-83]$ \\
\hline \multicolumn{2}{|l|}{ Primary } \\
\hline Colon/rectum & $67 / 63$ \\
\hline Nodal positive & 82 \\
\hline Nodal negative & 48 \\
\hline \multicolumn{2}{|l|}{ Liver metastases } \\
\hline Synchronous/metachronous & $77 / 53$ \\
\hline Surgical procedures & 167 \\
\hline Extended liver resection & 29 \\
\hline Major liver resection & 43 \\
\hline Minor liver resection & 95 \\
\hline Resection margin & 167 \\
\hline Ro & 161 \\
\hline $\mathrm{R} 1$ & 6 \\
\hline m-CS*, median (range) & $1(0-3)$ \\
\hline 0 & 22 \\
\hline 1 & 54 \\
\hline 2 & 44 \\
\hline 3 & 10 \\
\hline e-CS ${ }^{\star \star}$, median (range) & $1(0-4)$ \\
\hline 0 & 20 \\
\hline 1 & 51 \\
\hline 2 & 40 \\
\hline 3 & 15 \\
\hline 4 & 4 \\
\hline
\end{tabular}

*modified clinical risk score (consisting of primary tumor nodal status, size of CRLM $>5 \mathrm{~cm}$ and RAS mutation status); ${ }^{\star *}$ extended clinical risk score (consisting of primary tumor nodal status, size of CLM $>5 \mathrm{~cm}$ and mutation/alterations in the RAS/ RAF pathway and members of the SMAD family).

changes of 720 CGS genes in our study cohort. Depending on the frequency and previously reported clinical relevance in the setting of CRLM we focused on the gene alterations of APC, TP53, KRAS, NRAS, BRAF, SMAD2, SMAD3, SMAD4, PIK3CA and ERBB4 for further analyses (16).

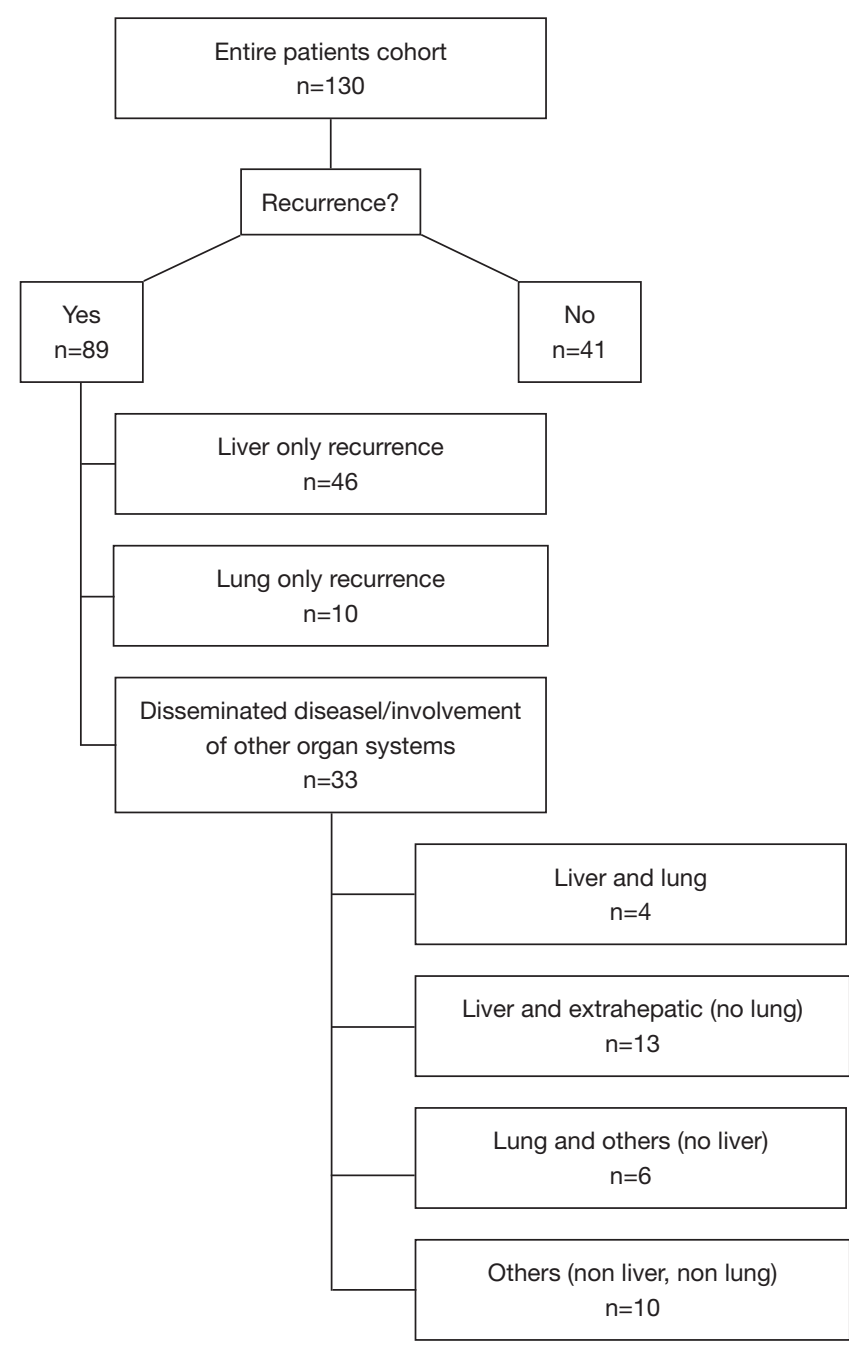

Figure 1 Patterns of recurrence.

The frequencies of gene alterations are listened in Table 2.

\section{Biological risk factors and their impact on recurrence}

First, we compared the distributional pattern of gene alterations with the oncological outcome and analysed patients with no recurrence $(n=41)$ versus patients with any kind of recurrence $(n=89)$. There were no significant differences detectable.

Going further into detail, we divided the recurrence group in 3 patterns of recurrence, in liver only, lung only and disseminated disease, respectively. Although some of the genes seemed to have prognostic influence, the multivariate Cox regression model did not reach significance (Table 3).

Within the next step we combined alterations and 
Table 2 Frequencies of alterations and mutations of cancer related genes

\begin{tabular}{lccc}
\hline \multirow{2}{*}{ Cancer-related gene } & \multicolumn{3}{c}{ Number of gene alterations and mutations } \\
\cline { 2 - 4 } APC & Total, $\mathrm{n}=130(\%)$ & No recurrence, $\mathrm{n}=41(\%)$ & Recurrence, $\mathrm{n}=89(\%)$ \\
TP53 & $111(85.4)$ & $37(90.2)$ & $74(83.1)$ \\
KRAS & $85(65.4)$ & $28(68.3)$ & $57(64.0)$ \\
PIK3CA & $39(30.0)$ & $13(31.7)$ & $26(29.2)$ \\
ERBB4 & $16(12.3)$ & $8(19.5)$ & $8(9.0)$ \\
SMAD4 & $10(7.7)$ & $4(9.6)$ & $6(6.7)$ \\
BRAF & $9(6.9)$ & $1(2.4)$ & $3(9.0)$ \\
NRAS & $8(6.2)$ & $3(7.3)$ & $5(5.6)$ \\
SMAD3 & $4(3.1)$ & $1(2.4)$ & $3(3.4)$ \\
SMAD2 & $4(3.1)$ & $0(0)$ & $4(4.5)$ \\
\hline
\end{tabular}

mutations of all cancer related genes in order to discover the impact of CGS on the prediction of recurrent disease. We found that gene alterations and mutations of the RAS/ RAF pathway and in members of the SMAD family had a significant predictive power for the time of recurrence after liver resection for CRLM $(\mathrm{P}<0.0001)$. Similarly, we found mutations of PIK3CA being associated with a better prognosis and a later occurrence of relapse $(\mathrm{P}=0.01)$.

Furtheron, we tried to identify biological risk factors to predict patterns of recurrence. Using a multivariate Cox regression model there was no significant mutation that could achieve significant predictive power (Table 3).

Finally, to draw more clinical relevance out of these findings, we developed a recurrence risk score (r-RS) by using multivariate Cox regression analyses, which enables a significant prediction of the time of recurrence. The score refers to mutations in the RAS/RAF pathway (1 point), in the SMAD family (1 point) and in PIK3CA. Mutations in PIK3CA were added as a positive vector ( -1 point) as it was associated with a later onset of recurrence and a significant better prognosis. The median recurrence-free survival in patients with a score of -1 was 634 days. In contrast, patients with a score of 2 (mutation in both the RAS/ RAF pathway and in the SMAD family but no mutation in PIK3CA) had a median time to recurrence of 85 days only. A score of 0 was associated with a median recurrence-free survival of 343 days (Figure 2).

The median overall $r$-RS of the complete study population was 0 (range, -1 to 2 ). The median $r$-RS and the range did not differ in the group with liver only recurrence and disseminated disease ( 0 , range, -1 to 2$)$. The group of lung only recurrence had a median r-RS of 1 (0 to 1 ) (Table 4$)$.

In the subgroup of the 6 patients with 2 and more repeated liver resection the median $\mathrm{r}$-RS was 0 (range, -1 to 0 ). There was no patient having a mutation in both the RAS/RAF pathway and in the SMAD family. One patient had a PIK3CA mutation (Table 5).

\section{Discussion}

In this study we analysed the impact of cancer related genes and their mutations on the incidence, the period of time of occurrence and the patterns of recurrence in 130 patients after curative resection of CRLM. The distribution of relapses in our cohort was similar as reported in other studies with an intrahepatic recurrence of $70 \%$ in total, and around $50 \%$ of them confined to the liver (6). This finding is of special interest because liver only recurrence may be suitable for liver directed therapies, e.g., for intraarterial chemotherapy or interventional treatment, but in particular also for repeated surgery. There are several reports about $2^{\text {nd }}, 3^{\text {rd }}$ or even $4^{\text {th }}$ hepatectomy for recurrent CRLM with survival data similar or even somewhat better than after initial resection $(9,17)$. In recent time promising results have been reported even after liver transplantation for irresectable CRLM. The rationale behind this concept is not least the assumption that in some patients the liver is, and probably will remain, the only site of recurrence. 
Table 3 Gene mutations and alterations in the group of recurrence

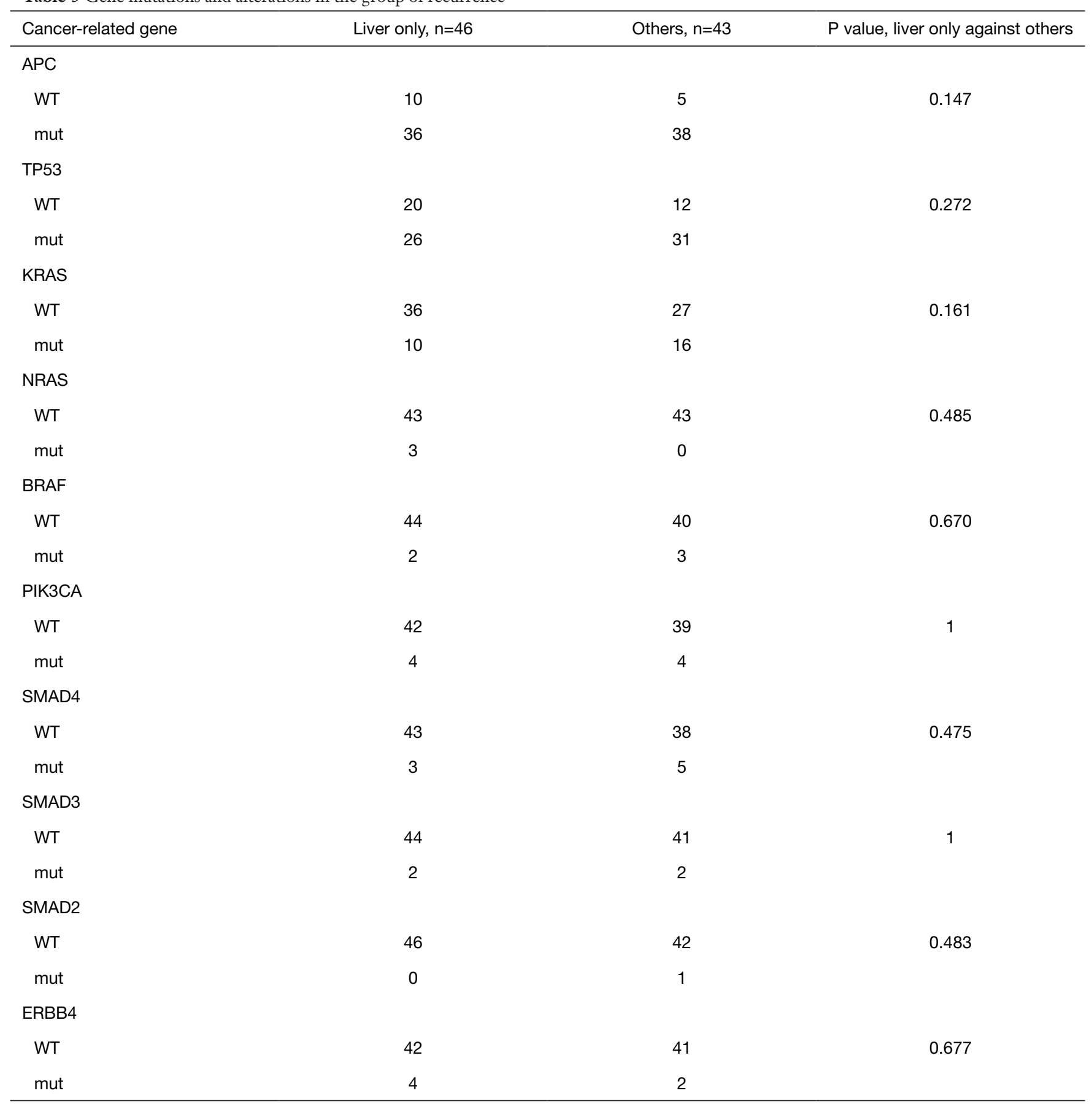

$P$ values of liver only against other organ systems and liver only against lung only and other organ systems.

Therefore, information on patterns of recurrence are of high clinical relevance. Unfortunately, our study did not find a correlation between mutations of cancer related genes and patterns of metastasising, in particular liver only recurrence could not be predicted. Even in the subgroup of 6 patients with repeated liver only recurrence no specific cluster of cancer gene alterations could be identified, except that in these patients there was neither a mutation in the RAS/RAF pathway nor in the SMAD family.

There are only few studies about mutation rates and 


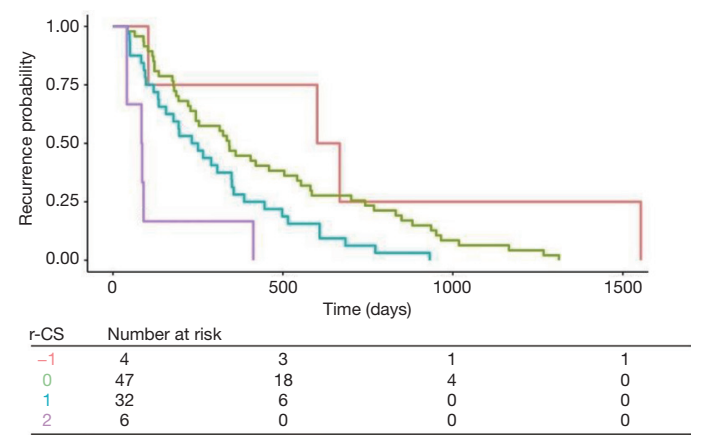

Figure 2 Recurrence free survival depending on mutations in the RAS/RAF pathway, the SMAD family and PIK3CA (recurrence risk score). patterns of extrahepatic recurrence. Some suggest a higher rate of extrahepatic relapse, especially of lung metastases after resection of KRAS mutated CRLM (18-21). Our findings are similar but do not show statistical significance probably due to the small number of patients in our cohort.

We could elaborate a score that correlates significantly with the risk of recurrence and especially with the risk of an early relapse within 100 days. We used the mutation status of RAS/RAF pathway and the SMAD family for this score. This is in accordance with previous findings that the mutation status of RAS and SMAD4 and even more of the RAS/RAF pathway and the entire SMAD family is superior

Table 4 Recurrence risk score and frequencies of mutations in the RAS/RAF pathway, SMAD family and PIK3CA in the group lung only recurrence $(\mathrm{n}=10)$

\begin{tabular}{lcccc}
\hline Patients with lung only recurrence & RAS/RAF pathway & SMAD family & PIK3CA & r-RS \\
\hline 1 & 1 & 0 & 0 & 1 \\
2 & 0 & 0 & 0 & 0 \\
3 & 1 & 1 & 0 & 2 \\
4 & 1 & 0 & 0 & 1 \\
5 & 1 & 0 & 0 & 0 \\
6 & 0 & 0 & 0 & 0 \\
7 & 0 & 0 & 0 & 1 \\
9 & 1 & 0 & 0 & 0 \\
10 & 0 & 0 & 0 & 0 \\
\hline
\end{tabular}

Table 5 Recurrence risk score and frequencies of mutations in the RAS/RAF pathway, SMAD family and PIK3CA in the group repeated resection for recurrent liver only metastases $(\mathrm{n}=6)$

\begin{tabular}{lccccc}
\hline Number of repeated liver resections & Patient & RAS/RAF pathway & SMAD family & PIK3CA & r-RS \\
\hline 2 & 1 & 0 & 0 & 0 & 0 \\
& 2 & 0 & 0 & -1 & 0 \\
3 & 3 & 0 & 0 & 0 & 0 \\
4 & 4 & 0 & 0 & 0 & 0 \\
\hline
\end{tabular}


to the mutation status of RAS alone in predicting prognosis after resection of CRLM (16,22).

In multivariable analysis, mutations that were associated with a better survival were observed in PIK3CA and TP53. These results confirm well with data reported by other authors (12). In our analysis the prognostic power of mutations of PIK3CA to predict recurrence was stronger than TP53 mutations. In order to keep the score easy, we focused on 3 factors only, two of them associated with worse prognosis (mutations in the RAS/RAF pathway and in the SMAD family) and one with better survival (mutations in PIK3CA).

Using this recurrence risk score (r-RS), ranging from -1 to 2 , we could identify a subgroup of patients (score 2 ) with a risk for very early recurrence (median time to recurrence nearly 3 months only) while in the group with mutations of PIK3CA only (score -1 ) the median time to recurrence was almost 2 years after resection. Noteworthy, the 6 patients with at least 2 and up to 4 repeated liver resections for liver only recurrence had a score of 0 or less.

The presented analysis has several limitations. In addition to its retrospective nature there is the small total number of 130 patients and only 89 of them having recurrent disease. Therefore, our findings need further validation in a larger cohort. This is in particular true for the groups with the lowest and the highest r-RS. Finally, the onset of recurrence may also be influenced by postoperative treatment. We did not further elucidate this since there is still ongoing discussion about the indication for adjuvant chemotherapy after resection of CRLM.

In conclusion, we could show that mutations in the RAS/RAF pathway and the SMAD family are risk factors for early recurrence and that mutations of PIK3CA and TP 53 are associated with a lower risk for recurrence after potentially curative resection of CRLM. Although our analysis failed to detect a correlation between patterns of mutations with patterns of recurrence we could develop a score that is predictive for the risk and timely onset of recurrence. The potential use of the risk score as additional criteria for adjuvant chemotherapy after potentially curative resection of CRLM needs further validation.

\section{Acknowledgments}

Funding: The study was in part supported by a research grant (to HL and MM) from MSD SHARP \& DOHME GMBH, Germany.

\section{Footnote}

Reporting Checklist: The authors have completed the STROBE reporting checklist. Available at https://dx.doi. org/10.21037/atm-21-292

Data Sharing Statement: Available at https://dx.doi. org/10.21037/atm-21-292

Conflicts of Interest: All authors have completed the ICMJE uniform disclosure form (available at https://dx.doi. org/10.21037/atm-21-292). HL received a research grant by MSD SHARP \& DOHME (MSD Germany). MM reports grants and non-financial support from EORTC, grants and non-financial support from AIO, grants and non-financial support from German Cancer Aid, grants and non-financial support from BMBF, during the conduct of the study; personal fees from Falk Foundation, personal fees from Lilly, grants and personal fees from MSD, personal fees from Roche, grants and personal fees from Pfizer, grants, personal fees and non-financial support from Amgen, grants, personal fees and non-financial support from Bristol-Myers Squibb, grants and personal fees from Merck Serono, personal fees from MCI Group, personal fees from Taiho, outside the submitted work. MK received a research grant of the Else-Kröner-Stiftung. The other authors have no conflicts of interest to declare.

Ethical Statement: The authors are accountable for all aspects of the work in ensuring that questions related to the accuracy or integrity of any part of the work are appropriately investigated and resolved. The study conforms to the provisions of the Declaration of Helsinki (as revised in 2013). The study was approved by the local ethic committee [837.141.13(8826-F)]. All patients signed an informed consent before participating the study.

Open Access Statement: This is an Open Access article distributed in accordance with the Creative Commons Attribution-NonCommercial-NoDerivs 4.0 International License (CC BY-NC-ND 4.0), which permits the noncommercial replication and distribution of the article with the strict proviso that no changes or edits are made and the original work is properly cited (including links to both the formal publication through the relevant DOI and the license). See: https://creativecommons.org/licenses/by-nc$\mathrm{nd} / 4.0 \%$. 


\section{References}

1. Jones RP, Jackson R, Dunne DF, et al. Systematic review and meta-analysis of follow-up after hepatectomy for colorectal liver metastases. Br J Surg 2012;99:477-86.

2. Rees M, Tekkis PP, Welsh FK, et al. Evaluation of longterm survival after hepatic resection for metastatic colorectal cancer: a multifactorial model of 929 patients. Ann Surg 2008;247:125-35.

3. Vauthey JN, Kawaguchi Y. Innovation and Future Perspectives in the Treatment of Colorectal Liver Metastases. J Gastrointest Surg 2020;24:492-6.

4. Folprecht G, Gruenberger T, Bechstein WO, et al. Tumour response and secondary resectability of colorectal liver metastases following neoadjuvant chemotherapy with cetuximab: the CELIM randomised phase 2 trial. Lancet Oncol 2010;11:38-47.

5. Bonnot PE, Passot G. RAS mutation: site of disease and recurrence pattern in colorectal cancer. Chin Clin Oncol 2019;8:55.

6. de Jong MC, Pulitano C, Ribero D, et al. Rates and patterns of recurrence following curative intent surgery for colorectal liver metastasis: an international multiinstitutional analysis of 1669 patients. Ann Surg 2009;250:440-8.

7. Torzilli G, Cimino MM. Extending the Limits of Resection for Colorectal Liver Metastases ENHANCED ONE STAGE SURGERY. J Gastrointest Surg 2017;21:187-9.

8. Abdalla EK, Vauthey JN, Ellis LM, et al. Recurrence and outcomes following hepatic resection, radiofrequency ablation, and combined resection/ablation for colorectal liver metastases. Ann Surg 2004;239:818-25; discussion 825-7.

9. Oba M, Hasegawa K, Shindoh J, et al. Survival benefit of repeat resection of successive recurrences after the initial hepatic resection for colorectal liver metastases. Surgery 2016;159:632-40.

10. Dueland S, Syversveen T, Solheim JM, et al. Survival Following Liver Transplantation for Patients With Nonresectable Liver-only Colorectal Metastases. Ann Surg 2020;271:212-8.

11. Buisman FE, Galjart B, van der Stok EP, et al. Recurrence Patterns After Resection of Colorectal Liver Metastasis are Modified by Perioperative Systemic Chemotherapy. World
J Surg 2020;44:876-86.

12. Chun YS, Passot G, Yamashita S, et al. Deleterious Effect of RAS and Evolutionary High-risk TP53 Double Mutation in Colorectal Liver Metastases. Ann Surg 2019;269:917-23.

13. Yamashita S, Chun YS, Kopetz SE, et al. Biomarkers in colorectal liver metastases. Br J Surg 2018;105:618-27.

14. Prasanna T, Karapetis CS, Roder D, et al. The survival outcome of patients with metastatic colorectal cancer based on the site of metastases and the impact of molecular markers and site of primary cancer on metastatic pattern. Acta Oncol 2018;57:1438-44.

15. Brudvik KW, Jones RP, Giuliante F, et al. RAS Mutation Clinical Risk Score to Predict Survival After Resection of Colorectal Liver Metastases. Ann Surg 2019;269:120-6.

16. Lang H, Baumgart J, Heinrich S, et al. Extended Molecular Profiling Improves Stratification and Prediction of Survival After Resection of Colorectal Liver Metastases. Ann Surg 2019;270:799-805.

17. Adam R, Bismuth H, Castaing D, et al. Repeat hepatectomy for colorectal liver metastases. Ann Surg 1997;225:51-60; discussion 60-2.

18. Vauthey JN, Zimmitti G, Kopetz SE, et al. RAS mutation status predicts survival and patterns of recurrence in patients undergoing hepatectomy for colorectal liver metastases. Ann Surg 2013;258:619-26; discussion 626-7.

19. Kemeny NE, Chou JF, Capanu M, et al. KRAS mutation influences recurrence patterns in patients undergoing hepatic resection of colorectal metastases. Cancer 2014;120:3965-71.

20. Tie J, Lipton L, Desai J, et al. KRAS mutation is associated with lung metastasis in patients with curatively resected colorectal cancer. Clin Cancer Res 2011;17:1122-30.

21. Yaeger R, Cowell E, Chou JF, et al. RAS mutations affect pattern of metastatic spread and increase propensity for brain metastasis in colorectal cancer. Cancer 2015;121:1195-203.

22. Kawaguchi Y, Kopetz S, Newhook TE, et al. Mutation Status of RAS, TP53, and SMAD4 is Superior to Mutation Status of RAS Alone for Predicting Prognosis after Resection of Colorectal Liver Metastases. Clin Cancer Res 2019;25:5843-51.
Cite this article as: Lang H, Baumgart J, Roth W, Moehler M, Kloth M. Cancer gene related characterization of patterns and point of recurrence after resection of colorectal liver metastases. Ann Transl Med 2021;9(17):1372. doi: 10.21037/atm-21-292 